\title{
Lack of Efficacy of High-Dose Intravenous Immunoglobulin Treatment of Severe Thrombocytopenia in Patients with Secondary Dengue Virus Infection
}

\author{
Efren M. Dimaano, Mariko Saito, Shoko Honda, Edna A. Miranda, Maria T. G. Alonzo, Myra D. Valerio, \\ Cynthia A. Mapua, Shingo Inoue, Atsushi Kumaori, Ronald Matias, Filipinas F. Natividad, and Kazunori Oishi* \\ Department of Internal Medicine and Virology, Institute of Tropical Medicine, Nagasaki University, Nagasaki, Japan; Department of \\ Disaster Prevention System, Faculty of Risk and Crisis Management, Chiba Institute of Science, Chiba, Japan; Laboratory for \\ Clinical Research on Infectious Diseases, International Research Center for Infectious Diseases, Research Institute for Microbial \\ Diseases, Osaka University, Osaka, Japan; Department of Blood Borne Diseases, San Lazaro Hospital, Manila, Research and \\ Biotechnology Division, St. Luke's Medical Center, Quezon City, The Philippines
}

\begin{abstract}
Because most cases of secondary dengue virus infection are associated with an increased level of plateletassociated IgG, a high dose of intravenous immunoglobulin (IVIG) may have an effect on the development of severe thrombocytopenia in this disease. A randomized, controlled study was conducted with two treatment groups consisting of a treatment (IVIG) group $(\mathrm{n}=15)$ and a non-treatment (non-IVIG) group $(\mathrm{n}=16)$ to determine whether a high dose of IVIG is effective in hastening the recovery from thrombocytopenia in patients with secondary dengue virus infection. No significant difference was found in the baseline demographic data between the two groups. No adverse effect of IVIG was observed, but no effect in hastening the recovery of platelet counts was found in patients with secondary dengue infections. The lack of efficacy of IVIG suggests that platelet clearance by macrophages through Fc $\gamma$ receptors is not a primary mechanism in this disease.
\end{abstract}

\section{INTRODUCTION}

Dengue virus types $1-4$ induce a wide spectrum of clinical manifestations, including hemorrhagic manifestations associated with thrombocytopenia and increased vascular permeability. Secondary infections, which are commonly observed in dengue-endemic areas, are more likely to constitute a risk factor for dengue hemorrhagic fever (DHF). ${ }^{1}$ Although dengue virus-induced bone marrow suppression decreases platelet synthesis, an immune mechanism of thrombocytopenia resulting in increased platelet destruction appears to be operative in patients with $\mathrm{DHF}^{2,3}$ This disease is now highly endemic in more than 100 tropical countries, and the number of cases has increased dramatically during the past three decades. ${ }^{3,4}$ More than 1,000 deaths occur annually due to DHF, and no specific treatment is currently available.

The high frequency of elevated platelet-associated IgG (PAIgG) in idiopathic thrombocytopenic purpura (ITP) suggests that PAIgG is involved in the mechanisms of thrombocytopenia. ${ }^{5,6}$ Platelets coated with IgG autoantibodies, which form PAIgG, undergo accelerated clearance through Fcy receptors that are expressed on tissue macrophages. Intravenous immunoglobulin (IVIG) is currently a widely accepted treatment option for ITP. Therapeutic activity of IVIG in the amelioration of ITP appears to involve the mechanism of competitive inhibition of activating Fc $\gamma$ receptors on phagocytic macrophages in the mononuclear phagocytic system by IVIG-sensitized erythrocytes. ${ }^{7}$ Recent studies demonstrated that the levels of PAIgG levels were inversely correlated with platelet count in patients in the acute phase of secondary dengue virus infections. ${ }^{8,9}$ An increased level of PAIgG was observed in $73.8-80.8 \%$ of patients with secondary dengue virus infection. These data indicate that the formation of PAIgG in patients with secondary dengue virus infection may result in thrombocytopenia due either to plate-

* Address correspondence to K. Oishi, Laboratory for Clinical Research on Infectious Diseases, International Research Center for Infectious Diseases, Research Institute for Microbial Diseases, Osaka University, Japan. E-mail: oishik@biken.osaka-u.ac.jp let clearance by macrophages or to platelet lysis. This disease can be classified as a dengue virus-induced ITP. It was hypothesized, therefore, that Fc $\gamma$ receptor blockade by a high dose of IVIG might inhibit the development of severe thrombocytopenia caused by secondary dengue virus infection. A previous case report had suggested this effect in a patient with dengue fever (DF). ${ }^{10}$

\section{MATERIALS AND METHODS}

Patients and study design. The present randomized, controlled study was conducted to determine the efficacy of a high dose of IVIG in hastening the recovery of platelets or inhibiting the development of severe thrombocytopenia in patients with secondary dengue virus infection. Thirty-six patients clinically suspected of being infected with dengue virus who fulfilled the inclusion criteria were admitted and enrolled in the study on the first day of admission (day 1) to San Lazaro Hospital (Manila, The Philippines) between October and November 2005. Dengue hemorrhagic fever was diagnosed according to World Health Organization (WHO) criteria. ${ }^{11}$ There were two inclusion criteria for these patients: 1 ) an acute phase of dengue illness (within 5 days after the onset of illness) verified by the particle agglutination test for dengue $\operatorname{IgM}^{12}$ and 2) severe thrombocytopenia (platelet count between $20,000 \mu \mathrm{L}$ and $80,000 / \mu \mathrm{L}$ ) without prominent manifestation of bleeding or shock. The exclusion criterion was a present history of platelet transfusion either before or after admission to the hospital. The study was reviewed and approved by the Bioethics Committees of San Lazaro Hospital and St. Luke's Medical Center. Parents or guardians of all patients provided written informed consent.

Of the patients enrolled, 34 were confirmed to be infected with the dengue virus infected on the basis of a positive result by IgM-capture enzyme-linked immunosorbent assay or reverse transcription-polymerase chain reaction. ${ }^{13,14}$ Three patients were diagnosed as having primary infections, and 31 patients were diagnosed as having secondary infections by a hemagglutination inhibition test. ${ }^{15}$ Laboratory tests were conducted at St. Luke's Medical Center (Quezon City, The Phil- 
ippines). Enrolled patients with secondary infections were randomly assigned to the IVIG group and non-IVIG treatment groups by means of sealed envelopes.

Treatment with IVIG. Human immunoglobulin $(2.5 \mathrm{~g} / \mathrm{vial}$, Gammamune; Bayer Health Care, Brea, CA) at a dose of 0.4 $\mathrm{g} / \mathrm{kg} /$ day was given intravenously to each patient in the IVIG group intravenously at a constant rate of $0.1 \mathrm{~g} / \mathrm{kg} / \mathrm{hour}$ on day 2 , and was continued each day until the fourth day after admission (day 4) for three days. The dose and frequency of IVIG in this study was chosen on the basis of previous investigations of IVIG to treat ITP. ${ }^{16,17}$ Standard treatment including intravenous fluids was given to all patients, regardless of group assignment, according to WHO guidelines. ${ }^{11}$

Evaluation. To determine the effects of a high dose of IVIG, patients in both groups were requested to remain hospitalized until the seventh day after admission (day 7). The primary measure of efficacy was an increase in platelets. Platelet counts in patients of both groups were monitored daily during hospitalization (days 1-7) and on day three after discharge (day 10) at an outpatient clinic. The target sample size could not be estimated for this study because no previous studies had determined the effects of IVIG on the thrombocytopenia during acute dengue virus infection. A interim target sample size of 30 was chosen to ensure that there would be at least a $70 \%$ chance for detecting a difference of $40 \%$ ( $100 \%$ versus $60 \%$ ), with a one-sided alpha level of 0.05 , in the frequency of platelet counts higher than $80,000 / \mu \mathrm{L}$ on the fourth day after admission in patients with dengue illness and a low platelet count between 20,000 and $80,000 / \mu L^{8,9}$

Statistical analysis. All the data are expressed as the mean \pm SD. Differences in the demographic and clinical data between the IVIG group and non-IVIG groups were tested using either a chi-square test or a Fisher's exact test for nominal variables. Differences in laboratory data between the IVIG and non-IVIG groups were analyzed using a Student's $t$-test for continuous variables. A $P$ value less than 0.05 was considered significant.

\section{RESULTS}

Demographic data. Thirty-one patients with secondary infection were randomized to either the IVIG group $(n=15)$ or the non-IVIG group $(\mathrm{n}=16)$ (Table 1$)$. Ten DF cases and

TABLE 1

Comparison of clinical features of patients with secondary dengue virus infection between the IVIG group and the non-IVIG group*

\begin{tabular}{lccc}
\hline \multirow{2}{*}{\multicolumn{1}{c}{ Parameter }} & \multicolumn{2}{c}{ Group } \\
\cline { 2 - 3 } & $\begin{array}{c}\text { IVIG } \\
(\mathrm{n}=15)\end{array}$ & $\begin{array}{c}\text { Non-IVIG } \\
(\mathrm{n}=16)\end{array}$ & $P$ \\
\hline Mean age, years (SD) & $16.3(3.6)$ & $14.3(3.4)$ & 0.108 \\
Male \% & 53.3 & 43.8 & 0.594 \\
Mean body weight, kg (SD) & $37.8(10.6)$ & $37.3(8.7)$ & 0.891 \\
$\begin{array}{l}\text { Days after onset (SD) } \\
\text { Laboratory data on day 1 }\end{array}$ & $3.9(0.6)$ & $3.7(1.1)$ & 0.462 \\
$\begin{array}{l}\text { Mean platelet count, } \\
\quad \times 10^{3} / \mu \text { L (SD) }\end{array}$ & $54.9(12.7)$ & $48.0(13.1)$ & 0.147 \\
$\quad$ Mean AST, U/L (SD) & $129.1(130.5)$ & $124.0(69.5)$ & 0.893 \\
$\quad$ Mean ALT, U/L (SD) & $100.6(105.2)$ & $59.5(31.5)$ & 0.146 \\
Days of severe & & & \\
$\quad$ thrombocytopenia (SD) & $3.1(1.0)$ & $2.5(0.8)$ & 0.11 \\
\hline * IVIG = intravenous immunoglobulin; AST $=$ aspartate aminotransferase; ALT $=$
\end{tabular}

*IVIG $=$ intravenous immunoglobulin; AST $=$ aspartate aminotransferase; ALT $=$
alanine aminotransferase. Period of severe thrombocytopenia indicates the days of platelet count between $20,000 \mu \mathrm{L}$ and $80,000 / \mu \mathrm{L}$.
5 DHF cases (1 DHF I and 4 DHF II) were assigned to the IVIG group, and $9 \mathrm{DF}$ cases and $7 \mathrm{DHF}$ cases (6 DHF II and 1 DHF III) were assigned to the non-IVIG group. The increase in the hematocrit (mean \pm SD) was significantly greater in DHF patients than in DF patients for both treatment groups $(30.0 \pm 6.3 \%$ versus $13.8 \pm 5.3 \%$ in the IVIG group and $29.9 \pm 12.9 \%$ versus $8.9 \pm 4.4 \%$ in the non-IVIG group; $P<0.001)$. No significant differences were found between the two groups with respect to demographic and laboratory data including peripheral platelet count and levels of aspartate aminotransferase and alanine aminotransferase at day 1.

Treatment with IVIG. Because the lowest platelet counts were found on day 2, the recovery phase, but not the phase of development for severe thrombocytopenia, was evaluated in these patients (Figure 1). Although patients who received IVIG were carefully monitored for adverse events, none were observed in patients in this group either during or after treatment. Despite treatment with a high dose of IVIG, no significant change in the platelet counts between day 2 (day of initiation of IVIG treatment) and day 7 was observed in either treatment group. Likewise, no difference was found in the duration of severe thrombocytopenia between the IVIG and non-IVIG groups (Table 1). No significant difference was found in platelet counts in the DF and DHF subgroups between the IVIG and the non-IVIG groups during the same period.

Levels of PAIgG also were examined in patients in both groups on days 2,5 , and 10 . The PAIgG levels (mean \pm SD $\mathrm{ng} / 10^{7}$ platelets) increased from baseline on day 2 in both the IVIG and non-IVIG groups (24.5 \pm 15 versus $33.8 \pm 28.6)$, decreased on day $5(18.5 \pm 8.4$ versus $13.7 \pm 5.2)$, and returned to normal levels $(11.0 \pm 7.8$ versus $8.7 \pm 3.7)$ on day 10 , which is consistent with our previous findings. ${ }^{9}$ No significant difference was found in levels of PAIgG in patients in the IVIG group and the non-IVIG group.

\section{DISCUSSION}

Although the patients were enrolled in an early phase of the illness (less than 4 days after onset), the effect of IVIG on

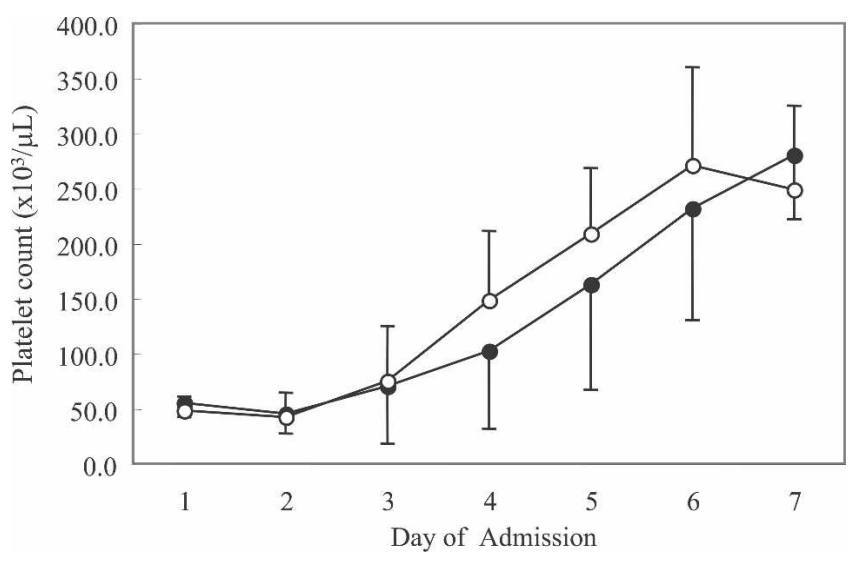

FIGURE 1. Comparison of platelet counts in patients with secondary dengue virus infections receiving a high dose of intravenous immunoglobulin (IVIG) and intravenous fluids (closed circle) and patients receiving intravenous fluids alone (open circle). The IVIG at a dose of $0.4 \mathrm{~g} / \mathrm{kg} /$ day was given to each patient in the IVIG group on the second, third, and fourth day after hospital admission. 
recovery of platelet count could be evaluated, but the inhibitory effect on the development of thrombocytopenia could not evaluated. No adverse effect of IVIG was noted, but there was no shortening of the time for platelet counts to return to normal levels in patients with secondary dengue infections. Because a rapid recovery of platelet counts is typically found in most patients with severe thrombocytopenia, no additional management of hemostatic abnormalities, including a high dose of IVIG, is required for such patients.

Our group recently developed an in vitro assay of phagocytosis of human platelets using flowcytometry. With this assay, it was shown that phagocytosis of platelets from patients with secondary infections by macrophages is significantly increased compared with that of platelets from healthy control subjects (Oishi K. and others, unpublished data). These data suggest that platelet clearance by macrophages plays a role in thrombocytopenia in this disease. Collectively, the lack of efficacy of IVIG in treating severe thrombocytopenia in secondary dengue virus infection shown in this study suggests that platelet clearance by macrophages through Fc $\gamma$ receptors is not a primary mechanism of thrombocytopenia in secondary dengue virus infection. Other immune mechanisms in this disease may involve platelet clearance by macrophages through complement receptor 3 (CR3) and complementmediated platelet lysis because complement activation mediated by circulating viral antigen is involved in the pathogenesis of this disease. ${ }^{6,18}$

Alternatively, de Castro and others recently conducted a pilot study to determine whether anti-D $\left(\mathrm{Rh}_{0} \mathrm{D}\right)$ immunoglobulin treatment, which resulted in a platelet increase of more than $70 \%$ in $\mathrm{Rh}+$ non-splenectomised patients with ITP, was effective in increasing platelet counts among pediatric and adult patients with dengue illness. ${ }^{19}$ Anti-D immunoglobulin also facilitates immune-mediated clearance of antibodycoated erythrocytes and spares sensitized platelets because of preferential destruction of erythrocytes by the mononuclear phagocytic system in ITP. ${ }^{7,20}$ Although de Castro and others demonstrated a trend toward higher platelet counts after treatment with anti-D immunoglobulin among such patients, there was no significant difference in the kinetics of platelet counts between pediatric and adult patients with dengue virus infection who received anti-D immunoglobulin and those who received placebo. ${ }^{19}$

In conclusion, the present study demonstrated a lack of efficacy for a high dose of IVIG in hastening the recovery of platelet counts in patients with secondary dengue virus infection. These data suggest that platelet clearance by macrophages through Fc $\gamma$ receptors is not a primary mechanism of thrombocytopenia in secondary dengue virus infection. Further studies are required to identify the immune mechanisms of thrombocytopenia in secondary dengue virus infection.

Received August 22, 2007. Accepted for publication August 28, 2007.

Acknowledgments: We thank Aruturo Cabanban and Eumella Salva and other staff of San Lazaro Hospital, and the staff of the Research Biotechnology Division, St. Luke's Medical Center.

Financial support: This study was supported by a Grant-in-Aid for Scientific Research (B: 16406029) from the Ministry of Education, Science and Culture, Japan and the 21st Century COE Program of Nagasaki University.

Authors' addresses: Efren M. Dimaano and Edna A. Miranda, Blood Borne Diseases, San Lazaro Hospital, Manila, The Philippines.
Mariko Saito, Shoko Honda, and Shingo Inoue, Department of Internal Medicine and Virology, Institute of Tropical Medicine Nagasaki University, 1-12-4 Sakamoto, Nagasaki 852-8523, Japan. Maria T. G. Alonzo, Myra D. Valerio, Cynthia A. Mapua, Ronald R. Matias, and Filipinas F. Natividad, Research and Biotechnology Division, St. Luke's Medical Center, 279 E. Rodriguez Sr. Boulevard, Cathedral Heights, Quezon City 1102, The Philippines. Atsushi Kumaori, Faculty of Risk and Crisis Management, Chiba Institute of Science, Choshi, Chiba 288-0025, Japan. Kazunori Oishi, Laboratory for Clinical Research on Infectious Diseases, International Research Center for Infectious Diseases, Research Institute for Microbial Diseases, Osaka University, 3-1 Yamadaoka, Suita, Osaka 565-0871 Japan, Telephone: 81-6-6879-4253, Fax: 81-6-6879-4255, E-mail: oishik@biken.osaka-u.ac.jp.

Reprint requests: Laboratory for Clinical Research on Infectious Diseases, International Research Center for Infectious Diseases, Research Institute for Microbial Diseases, Osaka University, 3-1 Yamadaoka, Osaka, 565-0871 Japan.

\section{REFERENCES}

1. Halstead SB, 1988. Pathogenesis of dengue: challenges of molecular biology. Science 239: 476-481.

2. La Russa VF, Innis BL, 1995. Mechanism of dengue virusinduced bone marrow suppression. Baillieres Clin Hematol 8: 249-270.

3. Oishi K, Saito M, Mapua CA, Natividad FF, 2007. Dengue illness: clinical features and pathogenesis. J Infect Chemother 13: 125133.

4. Gubler DJ, 2002. Epidemic dengue/ dengue hemorrhagic fever as a public health, social and economic problems in the 21st century. Trends Microbiol 10: 100-103.

5. McMillan R, 1981. Chronic idiopathic thrombocytopenic purpura. N Engl J Med 304: 1135-1147.

6. Cines DB, Blanchette VS, 2002. Immune thrombocytopenic purpura. N Engl J Med 346: 995-1008.

7. Crow AR, Song S, Siragam V, Lazarus AH, 2006. Mechanisms of action of intravenous immunoglobulin in the treatment of immune thrombocytopenia. Pediatr Blood Cancer 47: 710-713.

8. Oishi K, Inoue S, Cinco MT, Dimaano EM, Alera MT, Alfon JA, Abanes F, Cruz DJ, Matias RR, Matsuura H, Hasebe F, Tanimura S, Kumatori A, Morita K, Natividad FF, Nagatake T, 2003. Correlation between increased platelet-associated IgG and thrombocytopenia in secondary dengue virus infections. $J$ Med Virol 71: 259-264.

9. Saito M, Oishi K, Inoue S, Dimaano EM, Alera MTP, Robles MP, Estrella JR, Kumatori A, Moji K, Alonzo BMT, Buerano CC, Matias RR, Morita K, Natividad FF, Nagatake T, 2004. Association of increased platelet-associated immunoglobulins with thrombocytopenia and the severity of disease in secondary dengue virus infections. Clin Exp Immunol 138: 299-303.

10. Ascher DP, Laws HF, Hayes CG, 1989. The use of intravenous gammaglobulin in dengue fever, a case report. Southeast Asian J Trop Med Pub Hlth 20: 549-554.

11. World Health Organization, 1997. Dengue Haemorrhagic Fever: Diagnosis, Treatment, Prevention and Control. Second edition. Geneva: World Health Organization.

12. Berlioz-Arthaud A, Marfel M, Durand AM, Ogawa T, 2005. Evaluation of a new anti-dengue virus IgM particle agglutination kit in the context of the Pacific islands. World Health Organ Dengue Bull 29: 70-78.

13. Bundo K, Igarashi A, 1985. Antibody-capture ELISA for detection of immunoglobulin $\mathrm{M}$ antibodies in sera from Japanese encephalitis and dengue hemorrhagic fever patients. J Virol Methods 11: 15-22.

14. Morita K, Tanaka M, Igarashi A, 1991. Rapid identification of dengue virus serotypes by using polymerase chain reaction. $J$ Clin Microbiol 29: 2107-2110.

15. Clarke DH, Casals J, 1958. Techniques for hemagglutination and hemagglutination-inhibition with arthropod-borne viruses. $\mathrm{Am}$ J Trop Med Hyg 7: 561-573.

16. Kurlander RJ, Roose WF, 1986. Efficacy of a 2-day schedule for 
administering intravenous immunoglobulin (IVIg) in treating adults with ITP. Blood 291: 484.

17. Bierling P, Godeau B, 2005. Intravenous immunoglobulin for autoimmune thrombocytopenic purpura. Human Immunol 66: 387-394.

18. Avirutnan P, Punyanadee N, Noisaran S, Komoltri C, Thiemmeca S, Auethavornanan K, Jairungsri A, Kanlaya R, Tangtawornchailul N, Puttikhunt C, Pattnakisakul S, Yenchitsomnus P, Mongkolsapaya J, Kasinrerk W, Sittisombut N, Husmann M, Blettner M, Vasanawathana S, Bhakdi S, Mlasit P, 2006. Vascular leakage in severe dengue virus infections: a potential role for the nonstructural viral protein NS1 and complement. J Infect Dis 193: 1078-1088.

19. de Castro RA, de Castro JA, Barez M, Frias M, Dixit J, Genereux M, 2007. Thrombocytopenia associated with dengue hemorrhagic fever responds to intravenous administration of anti-D (Rh(0)-D) immune globulin. Am J Trop Med Hyg 76: 737-742.

20. Scaradavou A, Woo B, Woloski BM, Cunningham-Rundles S, Ettinger LJ, Aledort LM, Bussel JM, 1997. Intravenous anti-D treatment of immune thrombocytopenic purpura: experience in 272 patients. Blood 89: 2689-2700. 